\title{
(C) OPEN ACCESS \\ Variation and statistical reliability of publicly reported primary care diagnostic activity indicators for cancer: a cross-sectional ecological study of routine data
}

\author{
Gary Abel, ${ }^{1}$ Catherine L Saunders, ${ }^{2}$ Silvia C Mendonca, ${ }^{2}$ \\ Carolynn Gildea, ${ }^{3}$ Sean McPhail, ${ }^{4}$ Georgios Lyratzopoulos ${ }^{2,4,5}$
}

- Additional material is published online only. To view the files please visit the journal online (http://dx.doi. org/10.1136/bmjqs-2017006607).

${ }^{1}$ Primary Care, University of Exeter, Exeter, UK

${ }^{2}$ Cambridge Centre for Health Services Research, University of Cambridge, Cambridge, UK

${ }^{3}$ Knowledge and Intelligence Team (East Midlands), Public Health England, Sheffield, UK ${ }^{4}$ National Cancer Registration and Analysis Service, Public Health England, London, UK ${ }^{5}$ Epidemiology of Cancer Healthcare and Outcomes (ECHO) Group, Department of Behavioural Science and Health, University College London, London, UK

\section{Correspondence to} Dr Gary Abel, Medical School (Primary Care), University of Exeter, Exeter EX1 2LU, UK; g.a.abel@exeter.ac.uk

Received 17 February 2017 Revised 24 May 2017 Accepted 28 May 2017 Published Online First 28 August 2017

\section{Linked}

- http://dx.doi.org/10.1136/ bmjqs-2017-006782

CrossMark

To cite: Abel G, Saunders $C L$, Mendonca SC, et al.

BMJ Qual Saf 2018;27:21-30.
ABSTRACT

Objectives Recent public reporting initiatives in England highlight general practice variation in indicators of diagnostic activity related to cancer. We aimed to quantify the size and sources of variation and the reliability of practice-level estimates of such indicators, to better inform how this information is interpreted and used for quality improvement purposes.

Design Ecological cross-sectional study.

Setting English primary care.

Participants All general practices in England with at least 1000 patients.

Main outcome measures Sixteen diagnostic activity indicators from the Cancer Services Public Health Profiles. Results Mixed-effects logistic and Poisson regression showed that substantial proportions of the observed variance in practice scores reflected chance, variably so for different indicators (between 7\% and 85\%). However, after accounting for the role of chance, there remained substantial variation between practices (typically up to twofold variation between the 75 th and 25 th centiles of practice scores, and up to fourfold variation between the 90th and 10th centiles). The age and sex profile of practice populations explained some of this variation, by different amounts across indicators. Generally, the reliability of diagnostic process indicators relating to broader populations of patients most of whom do not have cancer (eg, rate of endoscopic investigations, or urgent referrals for suspected cancer (also known as 'two week wait referrals')) was high $(\geq 0.80)$ or very high ( $\geq 0.90)$. In contrast, the reliability of diagnostic outcome indicators relating to incident cancer cases (eg, per cent of all cancer cases detected after an emergency presentation) ranged from 0.24 to 0.54 , which is well below recommended thresholds $(\geq 0.70)$.

Conclusions Use of indicators of diagnostic activity in individual general practices should principally focus on process indicators which have adequate or high reliability and not outcome indicators which are unreliable at practice level.

\section{INTRODUCTION}

International variations in cancer survival have been attributed to healthcare system differences in primary care infrastructure. ${ }^{1}$ According to this hypothesis, in systems with a prominent primary care sector, 'gate-keeping' by general practitioners results in underutilisation of specialist investigations or referrals, leading to longer diagnostic intervals and poorer clinical outcomes for patients with cancer. ${ }^{2}$ Consequently, increasing attention is being paid to the role of primary care in diagnostic evaluation. ${ }^{3}$

In England, these considerations have led to the development of publicly reported diagnostic activity indicators for general practices (forming part of the Cancer Services Public Health Profile). ${ }^{4}$ Broadly, these include two principal families of indicators: some relating to broader populations of patients most of whom do not have cancer, for example, practice rates of urgent referrals for suspected cancer (hereafter referred to as 'two week wait' referrals) or endoscopy tests. Some other indicators relate only to incident cancer cases, for example, the proportion of all cancer diagnoses in a practice that were detected following 2-week wait referrals, otherwise known as 'detection rate.' For either class of measures we use the term activity (rather than 'performance') indicators, given lack of evidence on levels that represent best practice.

Information from the Cancer Services Public Health Profile is used to promote reflective practice, particularly following evidence suggestive of associations between the level of use of 2-week referrals and practice-level cancer survival. ${ }^{5} 6$ However, there are also concerns about the optimal use of practice-level 
Box 1 Inflation of observed variance by chance.

Where indicators are based on finite numbers, chance will inflate the apparent variation between units of observation. When indicators represent proportions, chance variation will be represented by the binomial distribution, whereas for rate indicators chance variation will be represented by the Poisson distribution. Just as we would not expect practices flipping 10 coins to all record precisely five heads (some will record more and some less by chance), we would not expect every practice with an underlying level of $50 \%$ to record exactly that. The effect of chance is larger when the numbers involved are small and it is this phenomenon which is often displayed in funnel plots where the 'funnel' is wider at the left of the graph where sample sizes are smaller. Where sample sizes are large, such that the role of chance is small and thus the statistical uncertainty on scores is small, the observed variation will closely approximate the underlying variation.

information on these indicators, given uncertainties about their statistical properties and the typically small number of new cancer cases annually in an average practice. $^{7}$ Where small numbers are involved there may be a considerable role of chance, leading to an artefactual inflation of the apparent variation such that, in some cases, most of the observed difference in practice activity may simply reflect chance (box 1). ${ }^{8}$

Against the above background, we characterised the size and sources of variation for diagnostic activity indicators included in the Cancer Services Public Health Profile, and the statistical reliability of potential comparisons of different practices using these measures.

\section{METHODS}

In summary, we analysed Cancer Services Public Health Profile data together with information on the age-sex composition of general practice populations to characterise and explain aspects of practice-level variation in diagnostic activity. After describing the observed variation for each diagnostic activity indicator, we used appropriate mixed-effects regression models to estimate the size of the underlying variation between practices. This underlying variation is that which would be seen if the effects of chance were not present. It can be thought of as the variation that would be seen if each practice had a very large sample size (see box 1). These models take into account the role of chance and additionally were used to account for differences in the make-up of practice populations. We further calculated the Spearman-Brown (interunit) reliability of each measure, to assess whether each diagnostic activity indicator included in the 'Profile' could reliably distinguish (by which we mean reliably rank or classify) general practices on the basis of their level of diagnostic activity. ${ }^{1}$

\section{Data}

We focused on Cancer Services Public Health Profile for 2013, the most recent year with available data at the time of the analysis. In general, practice indicators (table 1, see online supplementary appendix 1) comprise a 'numerator' (eg, the annual number of incident cancer cases, referrals or investigations in a practice), divided by an appropriate denominator (eg, the size of the patient population registered with the practice). Practices with $<1000$ registered patients are excluded from the public reporting scheme (which resultantly includes information for about $97 \%$ of all English general practices), and consequently our analyses.

We a priori categorised diagnostic activity indicators in those relating to broader populations of patients most of whom will not have cancer (diagnostic process indicators)—for example, endoscopy-investigated patients; and those relating to incident cases of cancer (diagnostic outcome indicators)—for example, the proportion of patients with cancer diagnosed after an emergency presentation—see also Background.

\section{Analysis}

After exclusions missing data were rare (see online supplementary appendix 3) and a complete case analysis was performed. For each indicator, the analysis comprised four steps.

Estimating the observed variation

To summarise the observed variation between general practices (ie, that seen in the publicly reported indicator data), we calculated the variance of the observed practice activity. In order to facilitate a meaningful comparison with modelled variance estimates described below, this variance was calculated on the $\log$ odds scale for proportion indicators and the log rate scale for rate indicators.

\section{Estimating modelled (underlying) variation}

To examine the degree to which observed variation reflects chance, we used a regression model including a random effect for general practice. These models estimate the underlying variance of practice activity (rather than that inflated by chance variation-where the additional chance variation would be described by the binomial or Poisson distributions). Mixed-effects logistic regression was used for 'proportion' indicators (eg, screening coverage) and mixed-effects Poisson regression for 'rate' indicators (eg, the rate of colonoscopy investigations, table 1). These models contained only a constant term and a random intercept for practice. Importantly, by using these models we are able to handle relatively sparse data (eg, from a single year of data collection) to partition the observed variation 
Table 1 Publicly reported diagnostic process or outcome cancer profile indicators during the study years (see also online supplementary appendices 1 and 3)

\begin{tabular}{|c|c|}
\hline Indicator name & Description (proportion or rate) \\
\hline \multicolumn{2}{|l|}{ Process indicators } \\
\hline Breast screening coverage & Per cent of the eligible practice population (women aged 50-69) screened in the last 36 months \\
\hline Cervical screening coverage & Per cent of the eligible practice population (women aged 25-64) screened in the target period \\
\hline Bowel screening coverage & Per cent of the eligible practice population (men and women aged 60-69) screened in the last 36 months \\
\hline Sigmoidoscopy rate* & Rate per 100000 registered patients per year \\
\hline Colonoscopy rate* & Rate per 100000 registered patients per year \\
\hline $\begin{array}{l}\text { Upper gastrointestinal endoscopy } \\
\text { rate* }\end{array}$ & Rate per 100000 registered patients per year \\
\hline TWW referral rate & Rate per 100000 registered patients per year \\
\hline TWW referral rate (colorectal) & Rate per 100000 registered patients per year \\
\hline TWW referral rate (lung) & Rate per 100000 registered patients per year \\
\hline TWW referral rate (skin) & Rate per 100000 registered patients per year \\
\hline TWW referral rate (breast) & Rate per 100000 registered patients per year \\
\hline \multicolumn{2}{|l|}{ Outcome indicators } \\
\hline TWW conversion rate & Per cent of TWW referrals resulting in a diagnosis of cancer \\
\hline TWW detection rate & Per cent of new cancer cases treated which resulted from a TWW referral \\
\hline Emergency route to diagnosi & Per cent of new cancer cases diagnosed via an emergency hospital admission \\
\hline Referred route to diagnosi & Per cent of new cancer cases diagnosed following outpatient referral to hospital \\
\hline Other route to diagnosis & Per cent of new cancer cases diagnosed through another route (eg, via screening) \\
\hline
\end{tabular}

into that attributable to chance and that which is underlying variation. These are estimates that are similar to those that would be obtained from observed scores aggregated over many years in a steady state. Practice activity was assumed to be normally distributed on the log odds scale for proportion indicators or log rate scale for rate indicators. Using the outputs of these models, we compared the variance of the modelled (underlying) practice diagnostic activity with that of observed practice scores (as above), to estimate the percentage of the observed variance (in the log odds or log rate scale, as applicable) that is attributable to chance.

Estimating modelled variation adjusted for the age-sex profile of practice populations

To examine how much of the observed variation reflects the age and sex make-up of practice populations we repeated the regression models additionally including variables representing proportions of practice populations in different age-sex strata (as we had 18 age groups, 35 such variables were used after excluding one age group-sex stratum which can be determined once all others are known and thus adds no information). From these 'adjusted-modelled' analyses, we estimated the variance of practice activity on the $\log$ odds or $\log$ rate scale as before, adjusted for the age and sex make-up of practice populations. This variance was compared with the variances for the observed and modelled activity to allow respective estimations of the proportion of the observed variance that is due to chance and practice population demography, and the proportion of the modelled variance that is due to practice population demography alone.

Distribution of practice activity

The variances estimated in all above three steps are hard to interpret in respect of the reported indicator values. For this reason, we plotted the distribution of indicator activity in the natural scale. Further, we estimated rate ratio or OR values (as applicable for rate and proportion indicators, respectively) comparing the 75 th with the 25 th, and the 90 th with the 10 th centiles of the distributions of practice activity for the observed, modelled and adjusted-modelled distributions. With the observed distribution we used observed centiles of the distribution to calculate these ratios, whereas for the modelled distributions and adjusted-modelled distributions a fully parametric estimation was made using the estimated variance of the random effect and respective percentiles of the normal distribution.

\section{Estimating reliability}

Finally, we used the same models as for estimating the underlying variation without adjustment for practice age-sex profile to estimate the Spearman-Brown (interunit) reliability of the reported practice activity. Briefly, reliability is a way to assess whether the measured activity for a particular organisation can be meaningfully distinguished from other organisations 
Box 2 Spearman-Brown (or interunit) reliability.

Spearman-Brown, or interunit, reliability (also known as rankability ${ }^{9-111524}$ ) is a measure of how reliably units (in this case general practices) can be distinguished (ranked, classified) from each other for a particular indicator. A value of 0 implies that all observed variability is due to chance and so units cannot be distinguished at all from each other on the basis of such an indicator. In contrast a value of 1 implies that all of the observed variability is real and thus all practices can be accurately classified. Reliability is defined as follows:

Reliability $=\frac{\text { underlying between unit variance }}{\text { underlying between unit variance }+\frac{\text { within unit variance }}{n}}$

where $\mathrm{n}$ is the number of observations for a given practice. Often reliability is thought of as the proportion of observed variance that is attributable to the underlying variance (alternatively, the proportion of overall variance not due to chance). However, in ascribing a single reliability value to all units we might suggest all have the same level of distinguishability. Reliability of binary and rate indicators depends on three factors:

- unit sample size, with a higher sample size leading to more precise unit score estimates and thus increasing reliability;

- unit score, with percentage scores closer to $50 \%$ leading to smaller within-unit variances on the log odds scale for the same sample size, thus leading to more precise unit score estimates and thus increasing reliability;

- between-unit variance, with larger between-unit variances making it easier to distinguish units with the same absolute precision of estimated score, thus increasing reliability.

Given variation in score and sample size between units we follow the example of Adams et al and consider reliability to be unit specific rather than a single value applying to the whole population. ${ }^{11}$ For this reason, the median reliability values shown in table 2 do not relate directly to the proportion of variance of observed activity attributable to chance in table 3 . The latter are heavily influenced by smaller practices with much larger variance due to chance.

(ie, whether high and low activity practices for a given indicator can be reliably distinguished from each other). ${ }^{9}$ Reliability is a term often used in USA and UK literature, but the same construct has also been denoted as 'rankability' in literature from continental Europe. Further details are included in box 2. A reliability coefficient (taking values from 0 -lowest reliability to 1 -perfect reliability) is estimated for each practice specific to its denominator and observed activity (see online supplementary appendix 2 for details). The median and IQR of practice reliability is then found along with the number of years of data required for $50 \%$ and $90 \%$ of practices to reach reliability thresholds of 0.7 and 0.9 . These thresholds are used following the examples of others, ${ }^{9-11}$ and while we recognise that any cut-off applied to such statistics is somewhat arbitrary, it has been suggested that the lower threshold (0.7) is desirable for any indicator of quality and the higher threshold (0.9) is preferable for higher stake applications such as pay for performance. $^{12} 10$

All analyses were performed in Stata V.13.1.

\section{RESULTS}

Between 7687 and 7954 practices were included in the analysis (table 2, see online supplementary appendix 3 ). The mean list size of these practices was 7034 (SD 4293) ranging from 1012 to 46126 . The median annual number of new cancer cases per practice was 28 (IQR 15-48). Diagnostic process indicators (relating to tested or referred patients) and diagnostic outcome indicators (relating to patients with incident cancer) had notably different number of cases or events per practice. For example, the median annual number of persons screened for colorectal cancer (relating to the 'bowel screening coverage' diagnostic process indicator) was 349 per practice; while the median annual number of cancer cases detected after 2-week wait referrals (relating to 'detection rate' diagnostic outcome indicator) was 12 per practice (table 2 ).

\section{Between-practice variation and the role of chance and that of} practice population demography

Overall the size of modelled (underlying) practice variation was substantial, with typically up to twofold variation between practices at the 75 th and the 25 th centiles of the distribution, and up to fourfold variation between practices at the 90th and 10th centiles (table 3, columns 7 and 10).

The proportion of overall variance in the observed activity that is due to chance ranged from $7 \%$ to $45 \%$ for diagnostic process indicators and from $65 \%$ to $85 \%$ for diagnostic outcome indicators (table 3, column 3). After accounting for variation due to chance, variation attributable to the age-sex profile of practice population ranged from $20 \%$ to $75 \%$ for diagnostic process indicators and from $5 \%$ to $31 \%$ for diagnostic outcome indicators (table 3, column 4). The combined proportion of variation due to both chance and practice age-sex profile ranged from $48 \%$ to $79 \%$ for diagnostic process and from $76 \%$ to $88 \%$ for diagnostic outcome indicators (table 3, column 5).

\section{Visualising variation}

In figures 1 and 2, we illustrate differences between the observed, modelled and adjusted-modelled variations for a diagnostic process indicator (the rate of 2 -week wait referrals) and an outcome indicator (the 


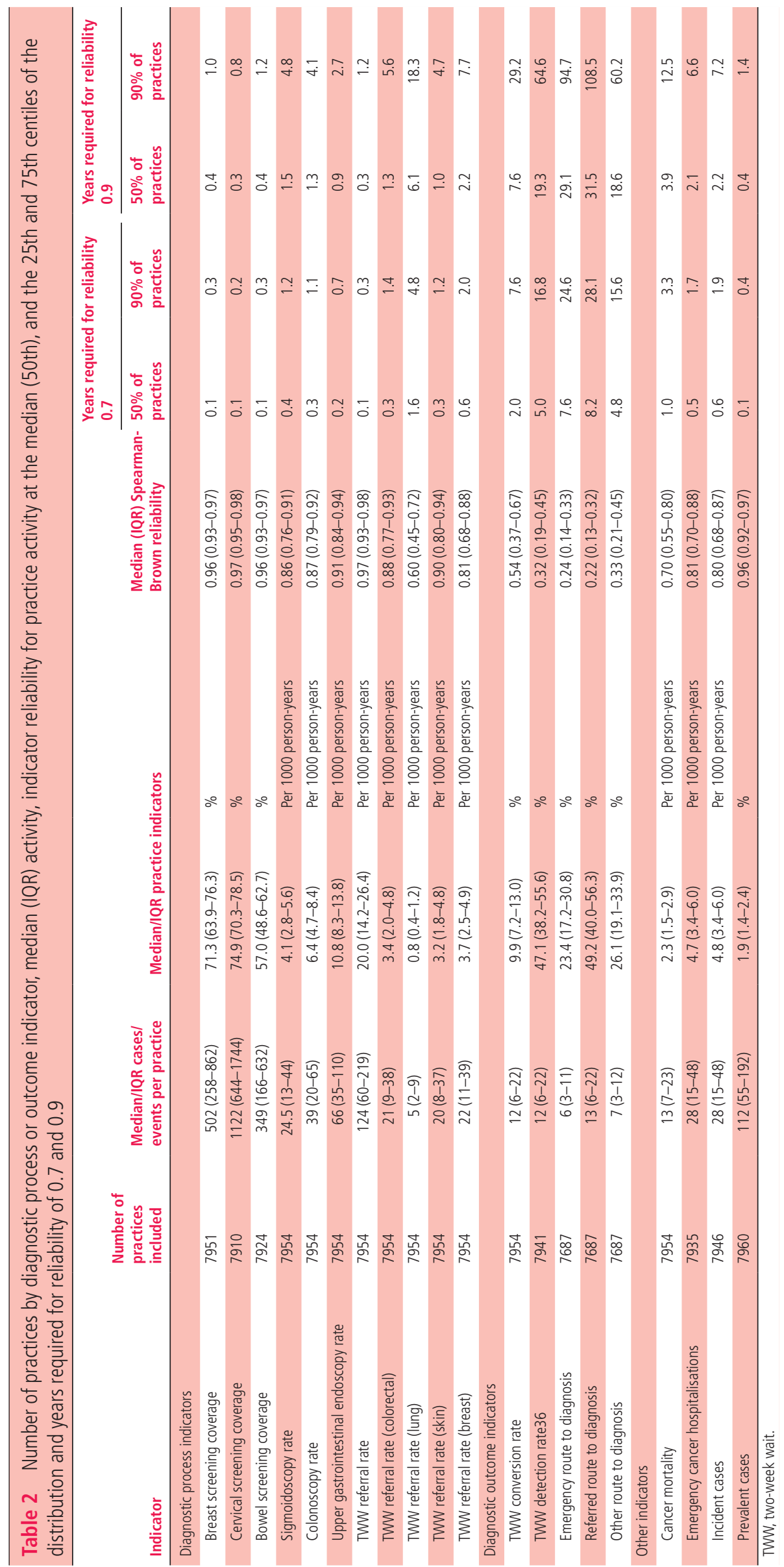




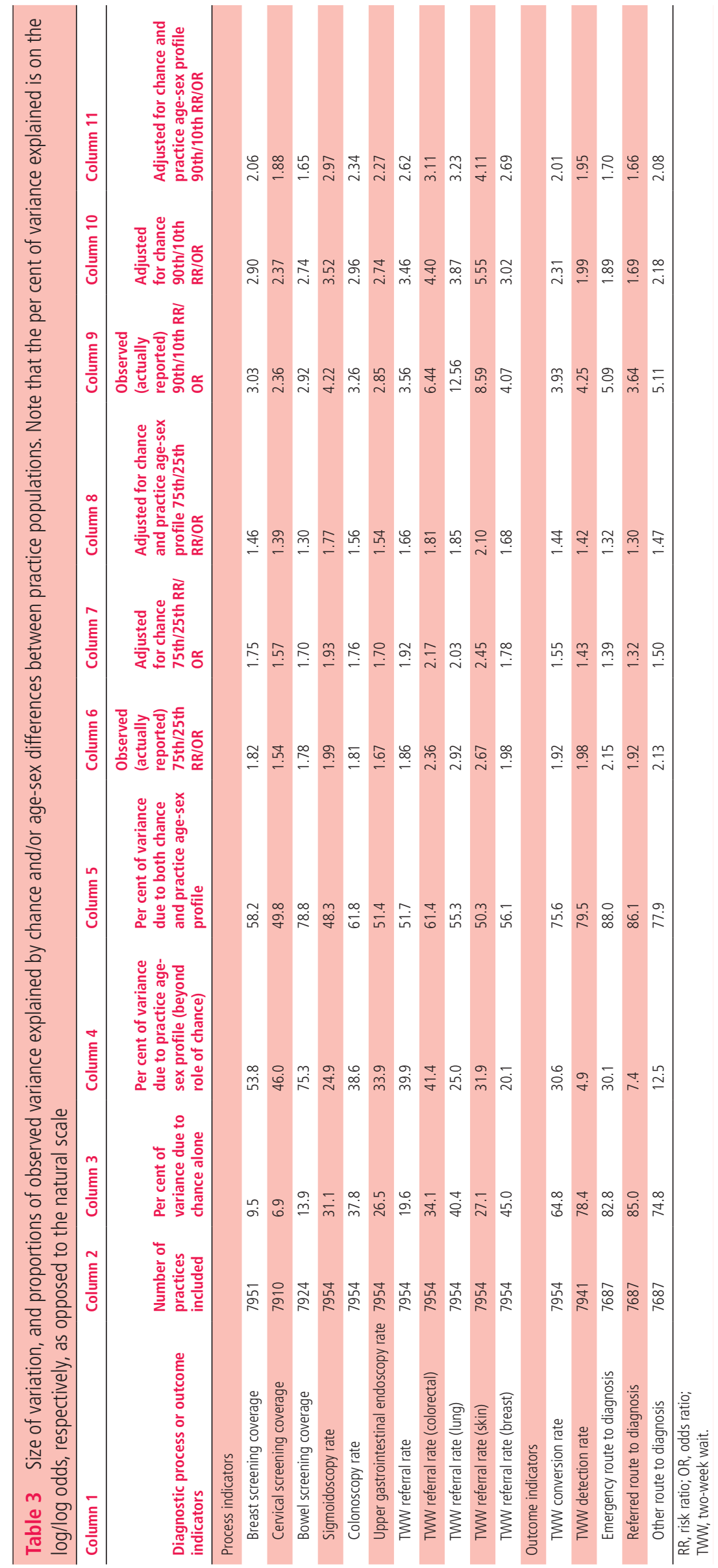




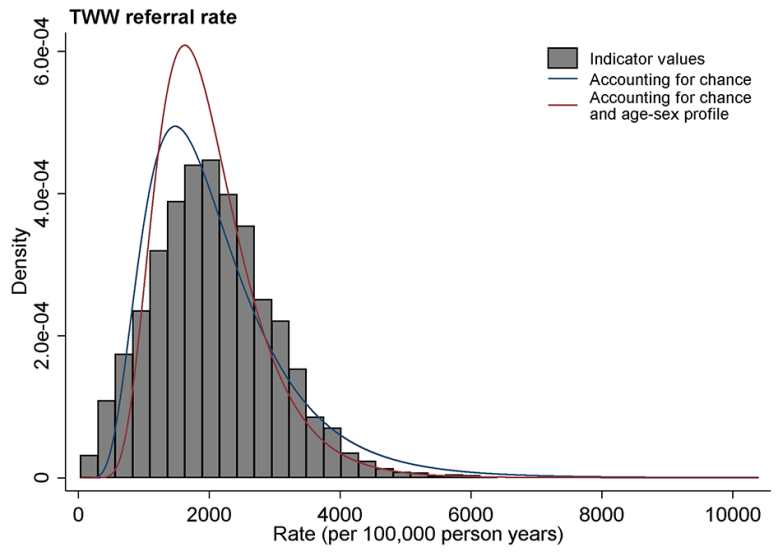

Figure 1 Illustration of the distribution of a diagnostic process indicator (2-week wait referral) for general practices. Observed values are denoted in grey histogram bars. The distribution of underlying practice activity, accounting for chance, is denoted with a blue curve line. The distribution of underlying practice activity adjusting for age-sex differences in practice populations is denoted by a red curve line. TWW, two-week wait.

proportion of all new cancer diagnoses in a practice diagnosed through an emergency presentation).

For 2-week wait referrals (figure 1), the spread of observed distribution (illustrated by the histogram) is similar to that of the modelled distribution accounting for chance (denoted by a blue line), although the latter is slightly narrower. This reflects the difference between observed and modelled variances for this indicator, which is $20 \%$, one of the smallest among all indicators (table 3, column 3).

Conversely, for the proportion of patients with cancer diagnosed as emergencies (figure 2), the spread of the observed variation is far wider than that of the modelled variation, indicating that the observed

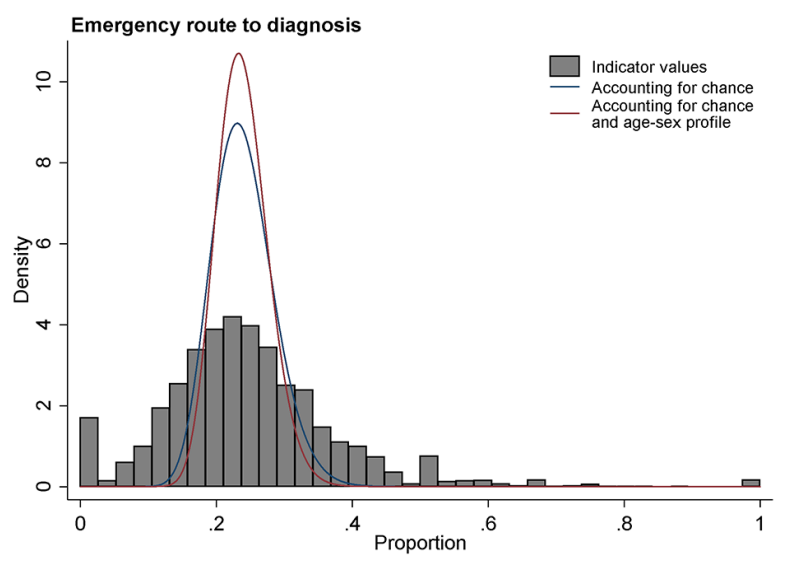

Figure 2 Illustration of the distribution of a diagnostic outcome indicator (per cent of cancer cases diagnosed following an emergency presentation) for general practices. Observed values are denoted in grey histogram bars. The distribution of underlying practice activity, accounting for chance, is denoted with a blue curve line. The distribution of underlying practice activity adjusting for age-sex differences in practice populations is denoted by a red curve line. differences are grossly inflated by chance. This also reflects that a very large proportion (ie, $83 \%$ ) of the observed variation for this indicator is due to chance (table 3, column 3). It is worth noting that the peaks in the observed distribution at 0 and 0.5 are dominated by practices with very few emergency presentations $(<5)$.

In both figure 1 and figure 2 the distributions shown by red lines (ie, accounting for both chance and the age-sex profile of practice population) are somewhat narrower than the distributions shown by blue lines (which simply account for chance). These differences reflect the $40 \%$ and $30 \%$ of variance beyond chance explained by the age-sex profile of practice in these two indicators, respectively (table 3, column 4).

\section{Indicator reliability}

The reliability of the publicly reported indicators included in the 'Cancer Services Public Health Profile' was bimodal: diagnostic outcome indicators relating to incident cancer cases (eg, rate of 2-week wait referrals, or the per cent of cancer cases presenting as emergencies) have low reliability, well below thresholds required for high-stake applications and performance management $(\geq 0.90)$ and, indeed, lower than thresholds of merely adequate reliability $(\geq 0.70)$. These include conversion (proportion of all 2-week wait referrals resulting in a cancer diagnosis) and detection rates (proportion of all new cancers treated which resulted from a 2-week wait referral), with median reliabilities of 0.54 and 0.32 , respectively (table 2 ). They also include the proportions of patients diagnosed via various routes (emergency, elective and 'other') which have median reliabilities of $\leq 0.33$. Reliable estimates could be achieved by using multiple years of data for these indicators, but often too many to make this practical (eg, more than 24 years to achieve a reliability of at least 0.7 for $90 \%$ of practices for the proportion of patients diagnosed via an emergency route, table 2).

This contrasts with diagnostic process indicators relating to broader populations (eg, patients investigated by endoscopy). Reliability is particularly high for all three screening coverage indicators $(r \geq 0.96)$; all three endoscopy activity indicators $(r \geq 0.86)$ and also for most 2-week wait referral indicators (any type $\mathrm{r}=0.97$, colorectal, breast and skin cancer, $\mathrm{r}>0.80$ for all three) (table 2). The single diagnostic process indicator with inadequate reliability is the 2 -week wait referral indicator for suspected lung cancer $(r=0.60)$ which, unusually among process indicators, on average relates to only few (5) cases per practice.

\section{DISCUSSION}

\section{Summary of main findings}

There is substantial variation between English general practices in respect of referral or investigation activity of relevance to cancer diagnosis, even after the roles of chance and the age-sex profile of practices are taken 
into account. However, practices can only be reliably classified for their propensity for high or low activity in respect of diagnostic process indicators (eg, 2-week referrals, endoscopy use or screening programme coverage). In contrast, diagnostic outcome indicators relating to incident cancer cases (eg, conversion or detection rates, or the proportion of cancers diagnosed as emergencies) are not reliable and practices should not be ranked in respect of such indicators. Reliability is driven by three factors: sample size, score and between-practice variance. In general, the poor reliability of diagnostic outcome indicators reflects the small sample size (see box 2). An exception to this general pattern is cancer mortality in a general practice population, which is an indicator with small numbers but modest reliability. It should be noted that mortality due to cancer in a general practice is a measure of disease burden and the relatively high reliability reflects larger variability between practices due to substantial sociodemographic differences in practice populations.

\section{Comparison with the literature}

We are not aware of prior studies specifically examining variation and reliability of a wide range of practice indicators included in the General Practice Profiles for Cancer. Observed variation in a small number of these indicators has been described previously, although previous studies have not formally examined the degree of variation due to mere chance. ${ }^{13}{ }^{14} \mathrm{~A}$ recent study from Scotland has attributed much of the variation in three indicators of referral activity (2-week wait referrals, and resultant conversion and detection rates) to 'random case-mix. ${ }^{7}$ In that paper, all chance variation is ascribed to variability in the case mix of presenting patients. ${ }^{7}$ If case mix was dominating the observed variation between practices then this could be overcome by adjusting or standardising for case mix. However, as shown by our study, chance variation would exist even in the absence of variable case mix. By examining a wide range of diagnostic evaluation measures included in our study (eg, regarding practice-level endoscopy use and screening programme coverage) and by quantifying the reliability of the indicators, we have shown that not all diagnostic activity indicators in current use are dominated by chance, and reporting could concentrate on those which are reliable. More broadly, our study adds to the prior literature on reliability and variability for organisational comparisons. Much of the prior literature relates to the context of hospital indicators where sample sizes are often larger. ${ }^{15} 16171819$ Previous studies described variation and/or the reliability of other indicators of general practice activity or quality chiefly considering patient experience, or prescription or cost patterns, but not diagnostic use indicators. ${ }^{9} 1020211122$ As has been remarked previously, the reliability of process measures is generally substantially higher than that of outcome measures, as the former encompass broader populations of patients with and without the disease or outcome of interest, whereas the latter relate to smaller populations of actual cases. ${ }^{23}$ Of course this is a generalisation, and it may be the case that outcome indicators are reliable, for example, a Dutch study found unintended reoperation after colorectal surgery to have an acceptable level of reliability. ${ }^{24}$

\section{Strengths and limitations}

We have used appropriate modelling techniques to separately account for the role of chance and the influence of differences in the age-sex make-up of practice populations, within the limits of the ecological data used. Our methods adjust for practice demography but do not account for the individual characteristics of patients who were referred or investigated. Individual level adjustment would have been preferable to fully adjust for confounding that may occur at patient level, but individual level case-mix data are not routinely collected as part of some of the respective data sets. ${ }^{25}$ By taking practice demography into account we may have overadjusted as, for example, patients of the same age may experience different diagnostic or referral activity in practices with averagely older populations.

Our reliability estimates appropriately relate to practice activity as it is publicly reported, which, with one exception, do not take into account individual case mix or the age-sex profile of practice populations. Where indicators are used for purposes of service planning or resource allocation, information is needed on observed as opposed to adjusted indicators. Where practice demography is the dominant source of variation, the reliability without adjustment for demography may simply reflect differences in the make-up of practice populations rather than real differences in clinical practice. In other words, such indicators may reliably distinguish practices with fewer older patients from those with more.

We have a priori not taken into account the deprivation scores of practice populations-because deprivation may be a mediator rather than a confounder of practice performance. We were not able to encompass practitioner-level variation in use of diagnostic tests or referrals-such a study is impossible to perform as data on diagnostic activity generated by individual doctors are not currently collected routinely (only data aggregated at practice level exist). However, other evidence indicates that the size of within-practice variation (ie, between the GPs of the same practice) is non-ignorable. ${ }^{26}$

\section{Implications}

Our study has several implications for policy and research. The fact that there is non-ignorable variation between practices (beyond that driven by chance and the demographic characteristics of the patient populations they serve) should motivate further inquiries 
about reasons likely to be responsible for this variation. This can take two approaches. First, studies that will examine potential correlations of diagnostic activity with other measures of practice quality and/or practice characteristics. Second, qualitative (including ethnographic and significant event audit) studies that will examine contextual and compositional (doctor or patient-related) factors determining diagnostic activity.

In their present form, 'Practice profiles' characterise practice scores reliably for some indicators but not others. These findings can be considered in respect of two possible applications. The first is one where all practices were ranked against each other for their position in the national distribution. As we have shown, for unreliable diagnostic outcome indicators such rankings will be dominated by chance and are unlikely to reflect underlying diagnostic activity. In contrast, reliable process indicators are well suited to such use. Second, individual practices can be compared with a national mean. Normally, uncertainty in practice estimates is taken into account when such comparisons are being made, and only practices where the difference from the national mean is statistically significant are flagged. While this obviates the problem of unreliably comparing practices against each other, there is still a high risk of type II error for unreliable indicators, meaning that many practices whose underlying activity is substantially different to the mean will not be identified as such. Several options exist to address the latter problem. For example, indicators with inadequate median reliability (ie, $<0.70$ ) can either be excluded from the reporting scheme, or be appropriately 'flagged' so that their interpretation and use (by healthcare professionals or managers, or members of the public) can be appropriately informed. The latter convention has been adapted by the public reporting of the 'Practice Profiles' recently (2015).

Evidence nonetheless indicates that greater use by general practices of 2 -week wait referrals, and greater use of upper gastrointestinal endoscopies are associated with better clinical outcomes. ${ }^{5}$ Examining potential associations between clinical outcomes and other types of diagnostic activity (other than 2-week wait referrals and upper gastrointestinal endoscopy) would be useful. Future research should also address the optimal levels of diagnostic or referral activity from the perspective of optimising population health outcomes and resource utilisation.

In conclusion, we identify substantial variation in practice level diagnostic processes and outcomes relating to cancer (beyond those that could be expected by chance or the age-sex profiles of practice populations), suggesting meaningful improvements are possible. Information on diagnostic outcome indicators has low reliability, therefore patients, clinicians and managers should avoid judging practices against these indicators. In contrast, diagnostic process indicators are statistically reliable and could be used to classify practice activity. The findings should motivate further research addressing the causes and consequences of variation in indicators of diagnostic evaluation in primary care. Although we examined practice indicators of diagnostic activity relevant to cancer, the findings could be relevant to other publicly reported primary care indicators, particularly if they relate to small number of cases per practice. Addressing the challenge of reliable measurement and public reporting of organisational or provider activity requires both further empirical work and a culture change among patients, practitioners and policymakers.

Contributors GL and GA conceived the study. CLS, SCM and CG performed the analysis with guidance from GA. GL, GA, CLS and SM drafted the original manuscript. All authors contributed to the interpretation of findings and revisions to the manuscript.

Funding This work was funded by Cancer Research UK (NAEDI) Grant No. C18081/A17854. The funder had no role in the study design; in the collection, analysis and interpretation of data; in the writing of the report; and in the decision to submit the article for publication. GL is supported by a Cancer Research UK Advanced Clinician Scientist Fellowship (C18081/A18180).

Competing interests All authors have completed the ICMJE uniform disclosure form at www.icmje.org/coi_disclosure. pdf and declare: support for the submitted work from Cancer Research UK; no financial relationships with any organisations that might have an interest in the submitted work in the previous 3 years; no other relationships or activities that could appear to have influenced the submitted work.

Patient consent The study was conducted using routinely collected and anonymous aggregated data which was made available in the public domain and as such consent from individual patients was not required.

Ethics approval This work was performed on data released by PHE under the Open Government Licence v2.0, as such no ethical review was necessary.

Provenance and peer review Not commissioned; externally peer reviewed.

Data sharing statement Data are available from the Public Health England Fingertips website http://fingertips.phe.org.uk/.

Open Access This is an Open Access article distributed in accordance with the terms of the Creative Commons Attribution (CC BY 4.0) license, which permits others to distribute, remix, adapt and build upon this work, for commercial use, provided the original work is properly cited. See: http://creativecommons.org/licenses/by/4.0/

(C) Article author(s) (or their employer(s) unless otherwise stated in the text of the article) 2018. All rights reserved. No commercial use is permitted unless otherwise expressly granted.

\section{REFERENCES}

1 Coleman MP, Forman D, Bryant H, et al. Cancer survival in Australia, Canada, Denmark, Norway, Sweden, and the UK, 1995-2007 (The International Cancer Benchmarking Partnership): an analysis of population-based cancer registry data. Lancet 2011;377:127-38.

2 Vedsted P, Olesen F. Are the serious problems in cancer survival partly rooted in gatekeeper principles? an ecologic study. $\mathrm{Br} \mathrm{J}$ Gen Pract 2011;61:508-12. 
3 Rubin G, Berendsen A, Crawford SM, et al. The expanding role of primary care in cancer control. Lancet Oncol 2015;16:1231-72.

4 Public Health England. Cancer services public health profile. http://fingertips.phe.org.uk/profile/cancerservices.

5 Møller H, Gildea C, Meechan D, et al. Use of the English urgent referral pathway for suspected cancer and mortality in patients with cancer: cohort study. BMJ 2015;351:h5102.

6 Shawihdi M, Thompson E, Kapoor N, et al. Variation in gastroscopy rate in English general practice and outcome for oesophagogastric cancer: retrospective analysis of Hospital Episode Statistics. Gut 2014;63:250-61.

7 Murchie P, Chowdhury A, Smith S, et al. General practice performance in referral for suspected cancer: influence of number of cases and case-mix on publicly reported data. $\mathrm{Br} \mathrm{J}$ Cancer 2015;112:1791-8.

8 Abel G, Lyratzopoulos G. Ranking hospitals on avoidable death rates derived from retrospective case record review: methodological observations and limitations. BMJ Qual Saf 2015;24:554-7.

9 Lyratzopoulos G, Elliott MN, Barbiere JM, et al. How can health care organizations be reliably compared?: lessons from a national survey of patient experience. Med Care 2011;49:724-33.

10 Roland M, Elliott M, Lyratzopoulos G, et al. Reliability of patient responses in pay for performance schemes: analysis of National General Practitioner Patient Survey data in England. BMJ 2009;339:b3851.

11 Adams JL, Mehrotra A, Thomas JW, et al. Physician cost profiling--reliability and risk of misclassification. N Engl J Med 2010;362:1014-21.

12 Rodriguez HP, von Glahn T, Chang H, et al. Measuring patients' experiences with individual specialist physicians and their practices. Am J Med Qual 2009;24:35-44.

13 Meechan D, Gildea C, Hollingworth L, et al. Variation in use of the 2-week referral pathway for suspected Cancer: a crosssectional analysis. Br J Gen Pract 2012;62:590-7.

14 Baughan P, Keatings J, O'Neill B. Urgent suspected cancer referrals from general practice: audit of compliance with guidelines and referral outcomes. Br J Gen Pract 2011;61:700-6.
15 Verburg IW, de Keizer NF, Holman R, et al. Individual and clustered rankability of ICUs according to Case-Mix-Adjusted Mortality. Crit Care Med 2016;44:901-9.

16 Guglielminotti J, Li G. Monitoring obstetric anesthesia safety across hospitals through multilevel modeling. Anesthesiology 2015; 122:1268-79.

17 Henneman D, van Bommel AC, Snijders A, et al. Ranking and rankability of hospital postoperative mortality rates in colorectal cancer surgery. Ann Surg 2014;259:844-9.

18 Krell RW, Hozain A, Kao LS, et al. Reliability of risk-adjusted outcomes for profiling hospital surgical quality. JAMA Surg 2014;149:467-74.

19 Shih T, Dimick JB. Reliability of readmission rates as a hospital quality measure in cardiac surgery. Ann Thorac Surg 2014;97:1214-8.

20 Stocks SJ, Kontopantelis E, Akbarov A, et al. Examining variations in prescribing safety in UK general practice: cross sectional study using the Clinical Practice Research Datalink. BMJ 2015;351:h5501.

21 Guthrie B, McCowan C, Davey P, et al. High risk prescribing in primary care patients particularly vulnerable to adverse drug events: cross sectional population database analysis in scottish general practice. BMJ 2011;342:d3514.

22 Sequist TD, Schneider EC, Li A, et al. Reliability of medical group and physician performance measurement in the primary care setting. Med Care 2011;49:126-31.

23 Mant J, Hicks N. Detecting differences in quality of care: the sensitivity of measures of process and outcome in treating acute myocardial infarction. BMJ 1995;311:793-6.

24 van Dishoeck AM, Lingsma HF, Mackenbach JP, et al. Random variation and rankability of hospitals using outcome indicators. BMJ Qual Saf 2011;20:869-74.

25 Saunders C, Abel G. Ecological studies: use with caution. Br J Gen Pract 2014;64:65-6.

26 Roberts MJ, Campbell JL, Abel GA, et al. Understanding high and low patient experience scores in primary care: analysis of patients' survey data for general practices and individual doctors. BMJ 2014;349:g6034. 